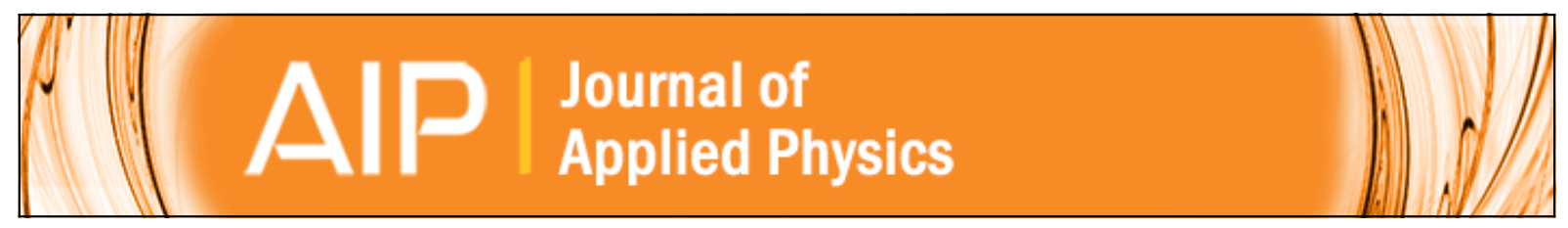

\title{
Weak localization and magnetoresistance of island-like thin copper films
}

Jianbiao Dai and Jinke Tang

Citation: Journal of Applied Physics 92, 6047 (2002); doi: 10.1063/1.1516869

View online: http://dx.doi.org/10.1063/1.1516869

View Table of Contents: http://scitation.aip.org/content/aip/journal/jap/92/10?ver=pdfcov

Published by the AIP Publishing

Articles you may be interested in

Magnetic properties of gadolinium substituted Bi2Te3 thin films

Appl. Phys. Lett. 102, 242412 (2013); 10.1063/1.4812292

Weak localization and antilocalization of hole carriers in degenerate $\mathrm{p}-\mathrm{Ge} 1-\mathrm{x} \mathrm{Mn} \times \mathrm{Te}$

J. Appl. Phys. 110, 113916 (2011); 10.1063/1.3669492

Weak localization and percolation effects in annealed In2O3-ZnO thin films

AIP Advances 1, 032149 (2011); 10.1063/1.3635375

Electron dephasing in wurtzite indium nitride thin films

Appl. Phys. Lett. 89, 232107 (2006); 10.1063/1.2400097

Spin-orbit interaction in thin bismuth films

Low Temp. Phys. 31, 326 (2005); 10.1063/1.1884436

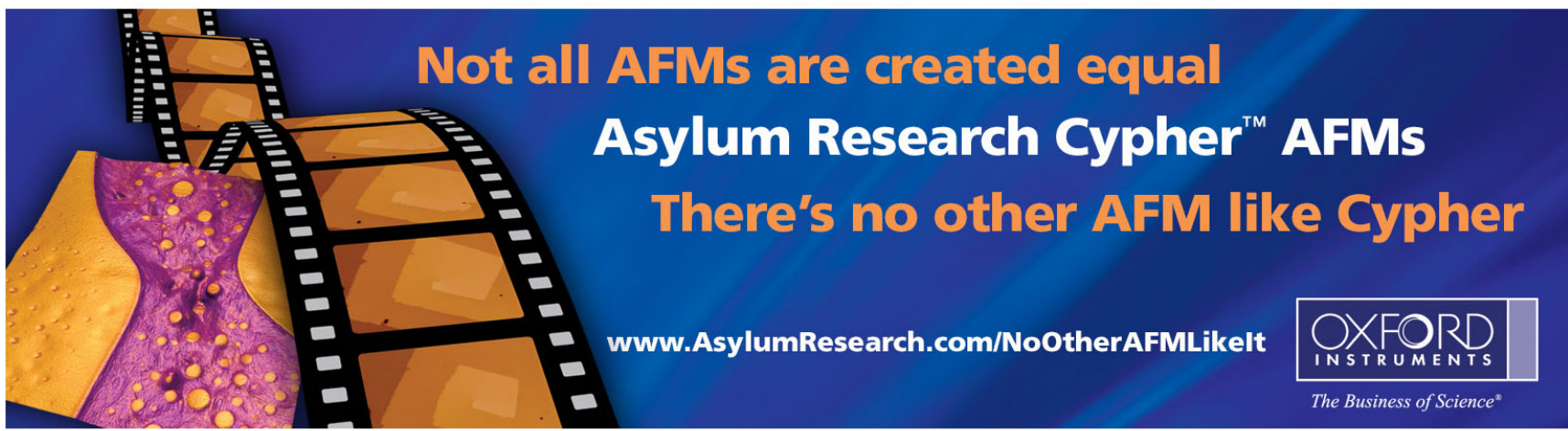




\title{
Weak localization and magnetoresistance of island-like thin copper films
}

\author{
Jianbiao $\mathrm{Dai}^{\mathrm{a})}$ and Jinke Tang ${ }^{\mathrm{b})}$ \\ Department of Physics, University of New Orleans, New Orleans, Louisiana 70148
}

(Received 16 April 2002; accepted 30 August 2002)

\begin{abstract}
We report magnetotransport and weak localization effects in island-like thin copper films. The samples show significant negative magnetoresistance (NMR) when a perpendicular magnetic field $\left(H_{\perp}>0.25 \mathrm{~T}\right)$ is applied. When the field is parallel to the film, the samples also show NMR for $H_{\|}>2.5 \mathrm{~T}$, below which positive magnetoresistance (PMR) is found. The temperature dependence of the resistivity and NMR in both perpendicular and parallel fields are due to the weak localization effect in the two-dimensional (2D) system. The observation of NMR in the parallel field is in agreement with Altshuler and Aronov's prediction [B. L. Altschuler and A. G. Aronov, Zh. Eksp. Teor. Fiz. Pis'ma Red. 33, 515 (1981)]. The PMR observed at low field is analyzed mainly in terms of the spin-orbit scattering, although other mechanisms may also be possible. It is suggested that the island-like geometry can significantly affect the localization and magnetotransport in 2D metallic films. (C) 2002 American Institute of Physics. [DOI: 10.1063/1.1516869]
\end{abstract}

\section{INTRODUCTION}

The conductivity of low dimensional systems does not display ordinary metallic properties, ${ }^{1-3}$ and scaling theories of localization have been applied in the analysis of twodimensional (2D) transport. There are two typical 2D electronic systems: ultrathin metallic films with disorder potential, and the near-surface inversion layers of semiconductors. ${ }^{4}$ An ultrathin metallic film can be a well defined quasi-2D system, which shows characteristic magnetoresistance (MR) and logarithmic temperature dependence of the resistivity. Several measurements have been performed on continuous pure films like $\mathrm{Cu},{ }^{5-7} \mathrm{Pt},{ }^{8} \mathrm{Au},{ }^{7,9} \mathrm{Ag},{ }^{7,10} \mathrm{Pd},{ }^{11}$ and indium oxidized. ${ }^{12}$ The results are in agreement with scaling theory, even though in some cases the increase of resistivity at low temperature is slower than that predicted. ${ }^{5}$

Negative magnetoresistance (NMR) has been predicted by the weak localization theory in $2 \mathrm{D}$ systems,${ }^{13,14}$ indicating that the localization effect could be destroyed when a magnetic field perpendicular to the film plane is applied. Previous experiments on metallic films ${ }^{5-11}$ have shown NMR in a perpendicular field due to the suppression of weak localization. It is known that the low dimension effects become significant when the thickness of a sample is less than the Thouless length $L_{T}=\left(2 l_{\mathrm{el}} l_{\mathrm{in}}\right)^{1 / 2}$, where $l_{\mathrm{el}}$ and $l_{\mathrm{in}}$ are the elastic and inelastic scattering lengths, respectively. When the Landau orbital size $(\hbar c / e H)^{1 / 2}$ becomes close to $L_{T}$, the localization effect can be suppressed by applying a perpendicular magnetic field. The threshold field is given as

$$
H_{c}^{\perp}=\hbar c / 2 e l_{\mathrm{el}} l_{\mathrm{in}},
$$

and the change in conductance is given by

\footnotetext{
a) Also at: Jiaotong University, Shanghai 200030, China.

b) Author to whom correspondence should be addressed; electronic mail: jtang@uno.edu
}

$$
\Delta \sigma_{\perp}=-\frac{e^{2}}{2 \pi^{2} \hbar}\left[\ln \left(\frac{\hbar c}{2 e H l_{\mathrm{el}} l_{\mathrm{in}}}\right)-\psi\left(\frac{1}{2}+\frac{\hbar c}{2 e H l_{\mathrm{el}} l_{\mathrm{in}}}\right)\right],
$$

where $\psi$ is the digamma function, and $H$ is the perpendicular field. In the case of heavy metals which have strong spinorbit scattering, e.g., in $\mathrm{Au}-\mathrm{Cu}$ films, ${ }^{5,7,15}$ the MR may change sign, and this will be discussed later.

Scaling theory suggests that there is no NMR in a strict 2D system when the field is applied parallel to the film. In 1984, Altshuler and Aronov ${ }^{16}$ predicted that, due to the finite thickness of a thin film, there is negative magnetoresistance in the parallel field $\left(\mathrm{NMR}_{\|}\right)$. The magnetoresistance in a parallel field is given by

$$
\Delta \sigma_{\|}=\frac{e^{2}}{2 \pi^{2} \hbar} \ln \left(\frac{t^{2} L_{T}^{2}}{12 L_{H}^{4}}+1\right),
$$

where $t$ is the film thickness, and $L_{H}=(\hbar c / e H)^{1 / 2}$ is the Landau orbit size. One sees that $\mathrm{NMR}_{\|}$will become zero when thickness $t=0$. Equation (3) can be understood by considering this is an orbital effect and $\mathrm{NMR}_{\|}$does not vanish until the film thickness is equal to zero. No significant parallel field $\mathrm{NMR}_{\|}$has been observed in previous experiments on homogeneous metallic films. According to Ref. 11, this is because of the following:

$$
\Delta \sigma_{\|} / \Delta \sigma_{\perp}=2 t^{2} / L_{T}^{2} \ll 1,
$$

where one can see the $\mathrm{NMR}_{\|}$is generally very small for ultrathin films. For the parallel field, the characteristic field strength is much stronger ${ }^{16}$ than field applied perpendicular to the film plane,

$$
H_{c}^{\|} \approx \frac{\hbar c}{e t \sqrt{l_{\mathrm{el}} l_{\text {in }}}} .
$$

The localization effect of highly inhomogeneous 2D systems of island-like films has not been extensively studied. There is very strong disorder in these systems and reduced 


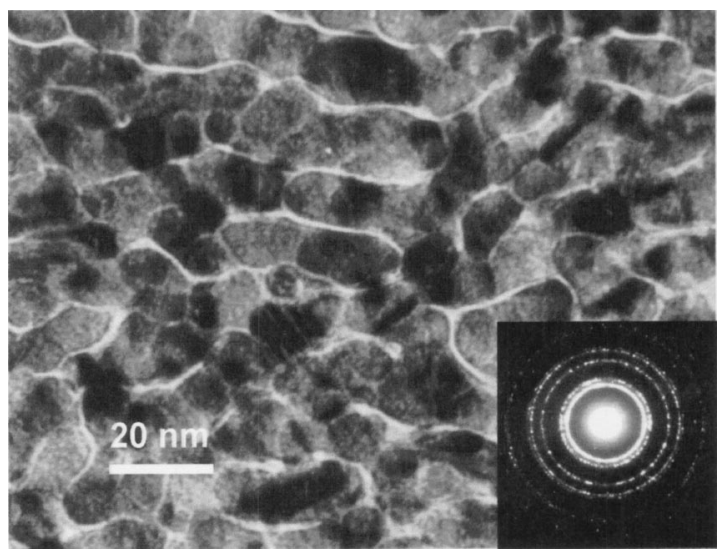

FIG. 1. TEM micrograph of the island-like $\mathrm{Cu}$ film of $50 \mathrm{~A}$ thickness. The inset is a diffraction pattern of the island-like $\mathrm{Cu}$.

conductance between islands. In addition, an island size effect may exist. Bergmann ${ }^{7}$ pointed out that weak localization may still exist in these systems. Here we report the observation of weak localization and unique MR effects in islandlike ultrathin $\mathrm{Cu}$ films. NMR has been observed in both perpendicular $\left(H_{\perp}\right)$ and parallel $\left(H_{\|}\right)$magnetic fields. We have found (1) significant $\mathrm{NMR}_{\perp}$ at perpendicular fields which follows $\ln H$ in the high field; (2) $\mathrm{NMR}_{\|}$which is close to the prediction given by Ref. 16; (3) positive MR (PMR) at low fields and a transition of the MR from positive to negative with an increase in field, pronounced in the case of parallel fields. An explanation of these MR properties will be given later by taking into account the weak localization and the influence of spin-orbit scattering. Compared to homogeneous films, it is suggested that the Thouless length and electron orbit size are affected by the geometry of the island-like structure.

\section{EXPERIMENTAL RESULTS}

$\mathrm{Cu}$ films were prepared by vacuum magneton sputtering deposition of pure $\mathrm{Cu}$ in an $\mathrm{Ar}$ atmosphere with pressure of $\sim 3$ mTorr onto a silicon wafer at room temperature. The background vacuum was $\sim 10^{-6}$ Torr. The sputtering rate was $2 \AA / s$ and the average sample thickness was $50 \AA$. Transmission electronic microscopy (TEM) was used to examine the morphology of the samples. Figure 1 shows the islandlike structure of $\mathrm{Cu}$ film which is neither continuous film nor totally isolated dots. The formation of this kind of film could be related to the Stranski-Krastanov mechanism. ${ }^{8,17}$ The average island size is about $15-20 \mathrm{~nm}$. Four-terminal dc resistivity measurements were performed using a Quantum Design physical properties measurement system (PPMS). The magnetotransport properties were also measured in a magnetic field from -9 to $9 \mathrm{~T}$, which was applied both perpendicular and parallel to the film. In the case of the parallel field, it was perpendicular to the direction of the current.

Figure 2 and the inset give the temperature dependence of the sample resistance per square. It increases with a decrease in temperature. The logarithmic dependence on the temperature is observed over $T<30 \mathrm{~K}$, which is in agreement with localization theory. MR in both the perpendicular

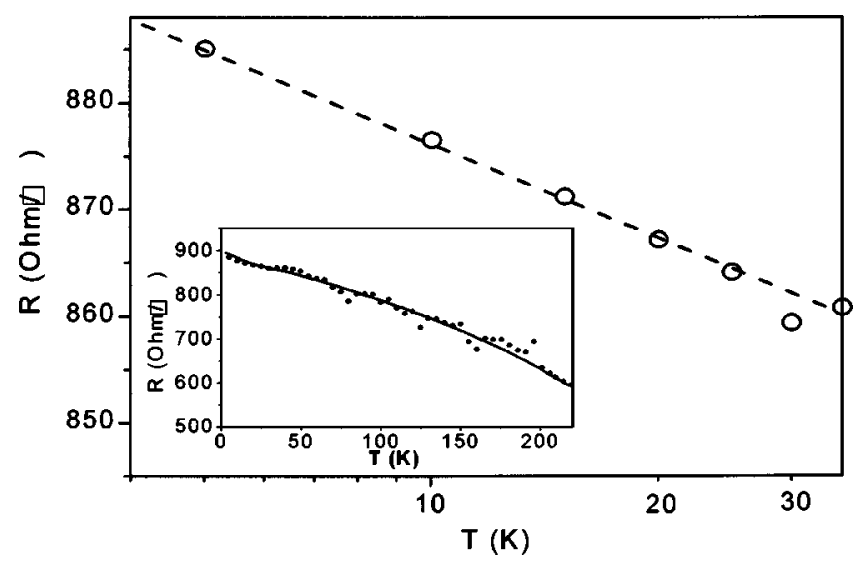

FIG. 2. Temperature dependence of the resistivity at low temperature (on a logarithmic scale). Inset: Resistivity over a wider temperature range.

and parallel magnetic fields was measured. Figures 3(a) and 3(b) show the resistance per square $R_{\square}$ vs $H_{\perp}$ and $H_{\|}$, respectively. In the perpendicular field the sample shows NMR when $H_{\perp}>0.25 \mathrm{~T}$. In low fields $\left(H_{\perp}<0.25 \mathrm{~T}\right)$, there is very small PMR as seen in the Fig. 3(a) inset. When the magnetic field is parallel to the film plane, the resistivity shows a abnormally large PMR when $H_{\|}<2.5 \mathrm{~T}$, changes to NMR when $H_{\|}>2.5 \mathrm{~T}$, and then monotonously decreases with an increase in $H_{\|}$.
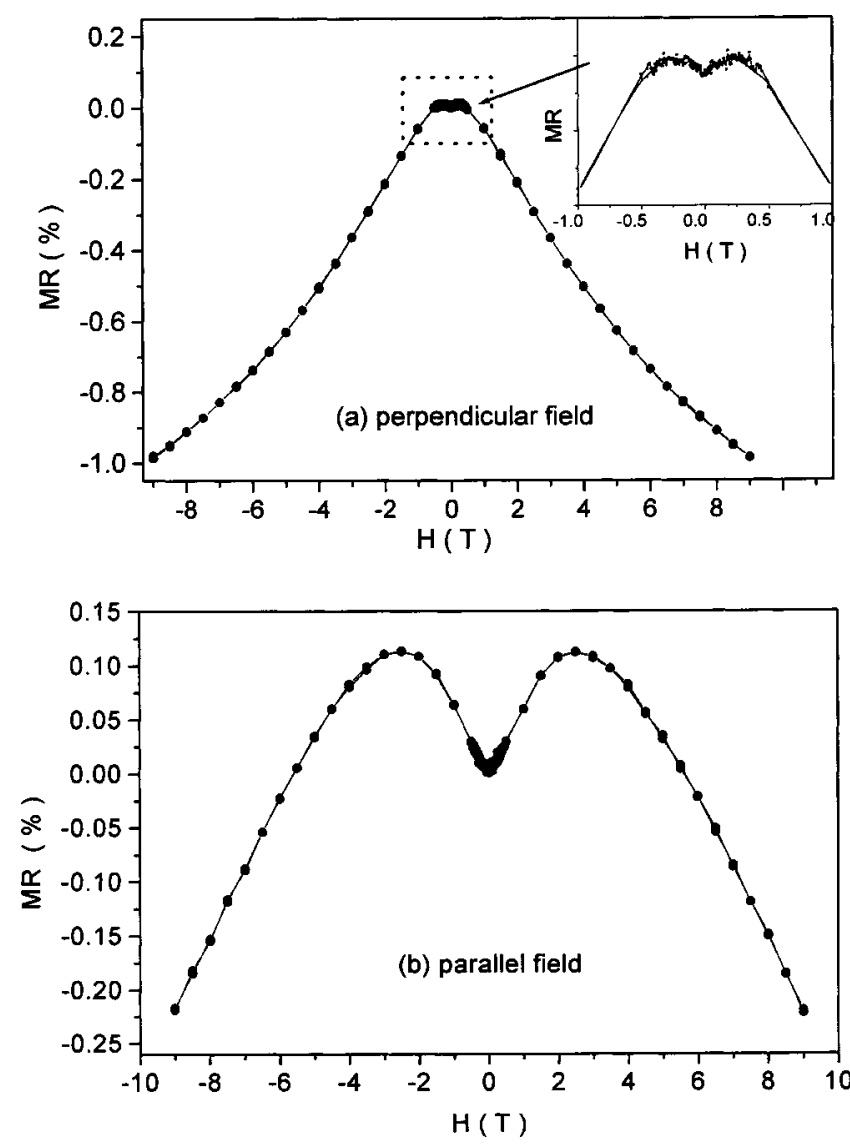

FIG. 3. (a) MR in the perpendicular field (b) MR in the parallel field. The data were obtained at $5 \mathrm{~K}$. 


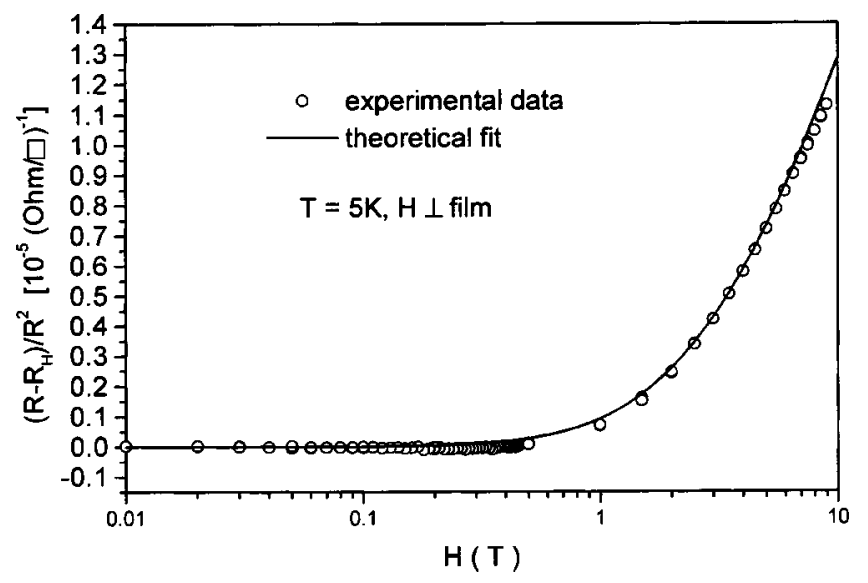

FIG. 4. Circles show the $\left(R-R_{H}\right) / R^{2}$ as a function of perpendicular field $H_{\perp}$ on a logarithmic scale. The solid line is the theoretical fit of NMR using Eq. (2), taking the value of $l_{\mathrm{el}} l_{\mathrm{in}}=5 \times 10^{4} \mathrm{~A}^{2}$.

\section{DISCUSSION}

In the following we discuss the MR observed in our experiments based on the weak localization effect and spinorbit scattering, and take into account the geometry effect of the island-like structure.

(i) $\quad$ The $\mathrm{NMR}_{\perp}$ of the island-like $\mathrm{Cu}$ films exhibits a well characterized weak localization effect. One can fit the MR data as a function of the field $H_{\perp}$ according to Eq. (2) quite well. The experimental data and theoretical fit are both shown in Fig. 4. When $H$ is significantly greater than $H_{\mathrm{c}}^{\perp}$, the NMR becomes linearly dependent on $\ln H$, which is typical of weak localization. It is also found that the value of $l_{\mathrm{el}} l_{\text {in }}$ is of the order of $10^{4} \mathrm{~A}^{2}$, in contrast to the typical $l_{\mathrm{el}} l_{\text {in }}$ values $\left(>10^{6} \mathrm{~A}^{2}\right)$ obtained from continuous $2 \mathrm{D}$ metallic films. ${ }^{5,7,11,12}$ The Thouless length $L_{T}$ of our films is smaller than that of the continuous films. This implies the average elastic scattering length $l_{\mathrm{el}}$ (and even $l_{\text {in }}$ ) is reduced by the geometry of the island-like structure of our $\mathrm{Cu}$ films due to strong scattering at the island boundary.

(ii) The $\mathrm{NMR}_{\|}$observed in our experiments provides strong evidence of the NMR predicted in Ref. 16 and expressed in Eq. (3). At $9 \mathrm{~T}, \mathrm{NMR}_{\|}$is as large as one fourth of $\mathrm{NMR}_{\perp}$. We believe the large $\mathrm{NMR}_{\|}$is due to the island-like structure since it has not been previously observed in homogeneous $\mathrm{Cu}$ films..$^{5-7} \mathrm{As}$ mentioned in (i), the Thouless length $L_{T}$ and effective Landau orbit size $L_{H}$ can be statistically reduced by the island-like structure. Since the ratio of $\Delta \sigma_{\|} / \Delta \sigma_{\perp}$ is determined by $2 t^{2} / L_{T}^{2}$ [Eq. (4)], a reduction in $L_{T}^{\frac{1}{2}}$ leads to a somewhat increased $\Delta \sigma_{\|}$. This provides a good explanation as to why the $\mathrm{NMR}_{\|}$was observed in our samples but not in previous experiments on homogeneous $\mathrm{Cu}$ films. That island-like geometry leads to relatively large $\mathrm{NMR}_{\|}$is also consistent with the experimental results of Komori et al., ${ }^{15}$ which have shown large $\mathrm{NMR}_{\|}$in highly inhomogeneous $\mathrm{Cu}-\mathrm{Cu}$ oxide mixed films although the mechanism was not explicitly given.

(iii) The PMR at low field which is found in both perpendicular $\left(\mathrm{PMR}_{\perp}\right)$ and parallel fields $\left(\mathrm{PMR}_{\|}\right)$is mainly due to the influence of spin-orbit scattering. PMR in 2D electron systems is usually explained by spinorbit scattering, ${ }^{7,11,18-21}$ which changes the sign of localization MR at low field for both $H_{\perp}$ and $H_{\|}$. In our experiment the $\mathrm{PMR}_{\perp}$ is relatively small, while the $\mathrm{PMR}_{\|}$is extraordinarily large.

At same time, the maximum PMR occurs at a much higher parallel field than perpendicular field. If one considers this maximum field as the characteristic field strength, our result is consistent with Eqs. (1) and (5). The field applied becomes effective in suppressing the weak localization at a much higher field for $H_{\|}$than for $H_{\perp},{ }^{16}$ which allows the $\mathrm{NMR}_{\|}$to increase to the measured value. It should be pointed out that the difference in MR peak field between parallel and perpendicular orientations is not due to the anisotropy of spin-orbit scattering. Rather, it is due to the difference in characteristic field strength of the suppression of the weak localization, expressed in Eqs. (1) and (5). The demagnetization effect cannot be a major contributing factor for the difference mentioned either. As a matter of fact, if the demagnetizing field were to play a role, the perpendicular orientation would exhibit a MR peak at a field higher than the parallel orientation because of the demagnetizing field associated with perpendicular orientation. To the contrary, the MR peak occurs at a parallel field 10 times greater than the perpendicular field. Additional mechanisms such as many electron interaction effects, magnetic impurity scattering and the Zeeman effect may be important in determining the $\mathrm{NMR}_{\|}$in our films. The fact that the $\mathrm{PMR}_{\perp}$ observed is much smaller than $\mathrm{NMR}_{\|}$could also be partially due to the much stronger contribution of NMR in the case of the perpendicular field, especially in low field range.

\section{CONCLUSIONS}

We have observed NMR and PMR due to the weak localization effect of a 2D electron system in island-like $\mathrm{Cu}$ thin films. The films show significant NMR in both the perpendicular and parallel fields, as well as PMR over a low field range. The NMR in the parallel field provides strong evidence for Altschuler and Aronov's theoretical prediction, and the NMR in the perpendicular field is consistent with weak localization theory. The PMR in the low field is mainly related to spin-orbit scattering, and the relative values of PMR can be qualitatively explained. This work also shows that the geometry of the thin films may significantly affect scaling of the electron localization and interactions, and that the island-like metallic films show some unique magnetotransport properties.

\section{ACKNOWLEDGMENTS}

The authors would like to thank Dr. L. Spinu for the transport measurements and Dr. W. Zhou and Dr. H. Xu for 
TEM micrographs. This work was supported by Sharp Laboratories of America and by Louisiana Board of Regents Support Fund Grant No. LEQSF(2000-03)-RD-B-10.

${ }^{1}$ D. J. Thouless, Phys. Rev. Lett. 39, 1167 (1977).

${ }^{2}$ E. Abrahams, P. W. Anderson, D. C. Licciardello, and T. V. Ramakrishnan, Phys. Rev. Lett. 42, 673 (1979).

${ }^{3}$ B. L. Altshuler, A. G. Aronov, and P. A. Lee, Phys. Rev. Lett. 44, 1218 (1980).

${ }^{4}$ D. J. Bishop, D. C. Tsui, and R. C. Dynes, Phys. Rev. Lett. 44, 1153 (1980).

${ }^{5}$ C. Van Hasendonck, L. Van den Dries, Y. Bruyn-Seraede, and G. Deutscher, Phys. Rev. B 25, 5090 (1982).

${ }^{6}$ L. Van den Dries, C. Van Haesendonck, Y. Bruyn-Seraede, and G. Deutscher, Phys. Rev. Lett. 46, 1149 (1981).

${ }^{7}$ G. Bergmann, Phys. Rep. 1, 107 (1984).

${ }^{8}$ R. S. Markiewicz and L. A. Harris, Phys. Rev. Lett. 46, 1149 (1981).
${ }^{9}$ N. Giordano and M. A. Pennington, Phys. Rev. B 47, 9693 (1993).

${ }^{10} \mathrm{~S}$. Hsu and J. M. Valles, Phys. Rev. B 74, 2331 (1995).

${ }^{11}$ R. S. Markiewicz and C. J. Rollins, Phys. Rev. B 29, 735 (1984).

${ }^{12}$ Z. Ovadyahu and Y. Imry, Phys. Rev. B 24, 7439 (1981).

${ }^{13}$ B. L. Altshuler, D. Khmel'nitzkii, A. I. Larkin, and P. A. Lee, Phys. Rev. B 22, 5142 (1980).

${ }^{14}$ S. Hikami, A. I. Larkin, and Y. Nagaoka, Prog. Theor. Phys. 63, 707 (1980).

${ }^{15}$ F. Komori, S. Kobayashi, and W. Sasaki, J. Phys. Soc. Jpn. 51, 3136 (1982)

${ }^{16}$ B. L. Altschuler and A. G. Aronov, Zh. Eksp. Teor. Fiz. Pis'ma Red. 33, 515 (1981) [JETP Lett. 33, 499 (1981)].

${ }^{17}$ E. Bauer and H. Poppa, Thin Solid Films 12, 167 (1972).

${ }^{18}$ H. Fukuyama, J. Phys. Soc. Jpn. 51, 1105 (1982).

${ }^{19}$ G. Deutscher and H. Fukuyama, Phys. Rev. B 25, 4298 (1982).

${ }^{20}$ P. A. Lee and T. V. Ramkrishnan, Phys. Rev. B 26, 4009 (1982).

${ }^{21}$ B. L. Altschuler, A. G. Aronov, A. I. Larkin, and D. E. Khmelnitskii, Zh. Eksp. Teor. Fiz. 81, 786 (1981) [Sov. Phys. JETP 54, 411 (1981)]. 